\title{
Impact of restrictions on parental presence in neonatal intensive care units related to coronavirus disease 2019
}

\author{
Ashley Darcy Mahoney ${ }^{1,2,3} \cdot$ Robert D. White $^{4} \cdot$ Annalyn Velasquez ${ }^{1,2} \cdot$ Tyson S. Barrett $^{5} \cdot$ Reese H. Clark $^{2}$. \\ Kaashif A. Ahmad ${ }^{2,6,7}$
}

(C) The Author(s), under exclusive licence to Springer Nature America, Inc. 2020

\begin{abstract}
Objectives To determine the relationship between the emergence of COVID-19 and neonatal intensive care unit (NICU) family presence as well as how NICU design affects these changes.

Study design A cross-sectional survey from April 21 to 30, 2020. We queried sites regarding NICU demographics, NICU restrictions on parental presence, and changes in ancillary staff availability.

Results Globally, 277 facilities responded to the survey. NICU policies preserving 24/7 parental presence decreased $(83-53 \%, p<0.001)$ and of preserving full parental participation in rounds fell $(71-32 \%, p<0.001)$. Single-family room design NICUs best preserved 24/7 parental presence after the emergence of COVID-19 (single-family room 65\%, hybriddesign $57 \%$, open bay design $45 \%, p=0.018)$. In all, $120(43 \%)$ NICUs reported reductions in therapy services, lactation medicine, and/or social work support.

Conclusions Hospital restrictions have significantly limited parental presence for NICU admitted infants, although singlefamily room design may attenuate this effect.
\end{abstract}

\section{Background}

Approximately $8.4 \%$ of United States newborns are admitted into neonatal intensive care units (NICUs) every year [1]. For extremely premature infants or those with major congenital anomalies, these admissions can last several months. Extended intimate contact of a newborn with

These authors contributed equally: Ashley Darcy Mahoney, Kaashif A. Ahmad.

Kaashif A. Ahmad

kaashif.ahmad@bcm.edu

George Washington University, Washington, DC, USA

2 MEDNAX Center for Research, Education, Quality, and Safety, Sunrise, FL, USA

3 Baptist Children's Hospital, Miami, FL, USA

4 Beacon Children's Hospital, South Bend, IN, USA

5 Utah State University, Logan, UT, USA

6 Baylor College of Medicine, San Antonio, TX, USA

7 Pediatrix Medical Group of San Antonio, San Antonio, TX, USA its parents, both mother and father, has been shown to have physiological and psychological benefits to both the newborn and it's parents [2]. This contact, often referred to as skin-to-skin or kangaroo care, provides many familiar sensory inputs such as the mother's voice, taste, and smell that cannot be replicated by others and improves physiological stability to the preterm infant [3]. This contact establishes a lasting bond that promotes both neurodevelopment in the infant [4] and enhanced mental health in the mother [3]. In addition to causing significant anxiety, acute stress, and post-traumatic stress for parents, admission to the NICU signifies a disturbance in the maternal-infant bond, which can be detrimental to the development of the newborn [4]. The introduction of family-centered care and allowing 24-h parental presence, especially in the setting of single-family rooms, may help increase parental presence, ameliorate some of these effects [5] and improve developmental outcomes [6].

In response to the emergence of Corona Virus Disease 2019 (COVID-19), the Centers for Disease Control and Prevention (CDC) issued a sequential series of recommendations. These included closure of elementary, middle, and high schools on 3/12/2020 [7], screening of visitors entering health care facilities on 3/20/2020 [8], and implementation 
of social distancing on 4/4/2020 which included working from home when possible [9]. While severe illness appears to be most prevalent in adults, transmission by asymptomatic adults and children may be widespread [10, 11]. Multiple media reports have documented prohibition of visitation for hospitalized adults, including when in extremis, in attempts to limit disease transmission [12, 13].

The tension between protection of infants and caregivers from serious infection and the desire to maximize the developmental outcome of newborns forces consideration of several options for restricted interaction, none of them optimal. While neonatal COVID-19 disease appears relatively uncommon, the risk-benefit calculation for restricting NICU access remains unclear. Further, no published data exist regarding the timing of implementing hospital and NICU entry restrictions or the extent of those restrictions after the emergence of COVID-19. We surveyed hospital sites globally to better understand the timeline and rigidity of hospital and NICU entry restrictions. In addition, we queried sites regarding new limitations in ancillary personnel such as therapy services and lactation consultants. We hypothesized that the availability of a large number of private ("single-family") rooms in a NICU would decrease the likelihood that the most restrictive parental presence policies would be considered necessary, when compared to NICUs in which most or all of the beds were in multibed rooms.

\section{Methods}

We performed a cross-sectional survey of global NICUs, with a focus on the United States, to determine hospital and NICU entry policies prior to and during the COVID-19 pandemic. The 21-item survey was built of both closed and open-response items requesting information on policies both before and during the spread of COVID-19 (Appendix). In particular, we requested information regarding location, type of hospital, NICU design, hospital and NICU entry policies, parental presence, and reductions in support staff. The survey was distributed through multiple venues from April 21 through April 30, 2020. These included an email to the American Academy of Pediatrics (AAP) Section of Neonatal-Perinatal Medicine listserv of NICU medical and quality improvement directors, MEDNAX practice medical directors, and to the MEDNAX Neonatology Forum. In parallel, we released the survey on Twitter, LinkedIn, Facebook, and group chats of neonatologists and neonatal nurse practitioners. In all venues we requested a response from the NICU medical or nursing director.

We included only those responses with at least completion of the first two sections of the survey (NICU demographics and visitation practices) and excluded multiple responses from the same NICU. To evaluate duplicate responses, we examined NICU zip code and baseline demographics. We determined a priori to include the first fully completed survey from any NICU or, in the case of multiple partially completed surveys, the first submitted response. While the stated preference was for NICU medical or nursing directors to respond to the survey, due to the methods of distribution this could not be controlled for.

The primary outcomes of interest were the presence of policies allowing 24-h parental presence in the NICU and allowing full parental participation in NICU rounds. Secondary outcomes of interest included screening policies related to hospital entry and the impact of COVID-19 on NICU staffing availability. The study was approved by the Methodist Healthcare Institutional Review Board, San Antonio, TX.

\section{Data analysis}

Two main approaches were used to analyze the data: sample descriptive statistics and comparisons of policies from before the spread of COVID-19 to after the start of the spread. For descriptive statistics, we used counts and percentages, presented in tables and figures. For policy comparisons, given the survey responses were all categorical, we used a series of McNemar's tests for all pre-post comparisons and Chi-square tests for comparisons between NICU designs. To adjust for the multiple hypothesis tests, we used a false discovery rate adjustment throughout this manuscript [14]. Analyses were performed in SPSS version 26 and $\mathrm{R}$ version 3.6.2.

\section{Results}

A total of 339 responses were received. Of these, 56 were excluded for incompletion of at least the first two survey pages and 6 excluded for duplicate NICU responses, leaving 277 surveys for analysis. Respondents most frequently learned of the survey through AAP and MEDNAX email requests (83\%) and responding NICUs were largely in the United States (91\%) with Texas, California, and Florida NICUs comprising $33 \%$ of the total cohort (Table 1, Fig. 1a). The characteristics of the responding NICUs are described in Table 1.

Across the United States, the timeline for implementing state-wide stay at home orders did not begin until the second half of March [15]. However, hospital and NICU entry policies began to change in the first week of January with a rapid increase in hospitals adopting policy changes throughout March, the majority prior to the issuance of CDC guidance (Fig. 2). At the state level, the mean date for changing hospital entry policies varied significantly. 
Table 1 Demographics of responding NICUs.

\begin{tabular}{|c|c|}
\hline NICU characteristic & $n=277$ \\
\hline NICU level & No $(\%)$ \\
\hline Level $1(n, \%)$ & $2(1)$ \\
\hline Level $2(n, \%)$ & $19(7)$ \\
\hline Level $3(n, \%)$ & $157(57)$ \\
\hline Level $4(n, \%)$ & $99(36)$ \\
\hline \multicolumn{2}{|l|}{ NICU design } \\
\hline Open ( $\geq 90 \%$ of beds in rooms of $4+$ beds, $n, \%)$ & $134(48)$ \\
\hline Hybrid $(n, \%)$ & $67(24)$ \\
\hline Single-Family Room ( $\geq 90 \%$ of beds in SFR, $n, \%$ ) & $76(27)$ \\
\hline \# of NICU beds (median, IQR) & $34(20,55)$ \\
\hline \multicolumn{2}{|l|}{ Type of unit } \\
\hline NICU, Outborn only $(n, \%)$ & $21(8)$ \\
\hline NICU, Inborn only $(n, \%)$ & $62(22)$ \\
\hline NICU, Inborn and Outborn $(n, \%)$ & $194(70)$ \\
\hline Located Within the United States (\%) & $251(91)$ \\
\hline \multicolumn{2}{|l|}{ Most represented States } \\
\hline Texas $(n, \%)$ & $41(15)$ \\
\hline California $(n, \%)$ & $30(11)$ \\
\hline Florida $(n, \%)$ & $22(8)$ \\
\hline New York $(n, \%)$ & $13(5)$ \\
\hline Pennsylvania $(n, \%)$ & $10(4)$ \\
\hline \multicolumn{2}{|l|}{ Most represented non-US countries } \\
\hline Saudi Arabia & $6(2)$ \\
\hline United Kingdom & $4(1)$ \\
\hline Spain & $4(1)$ \\
\hline Canada & $3(1)$ \\
\hline France, India & $2(1)$ \\
\hline \multicolumn{2}{|l|}{ Where did you hear about the survey? } \\
\hline American Academy of Pediatrics e-mail & $104(38)$ \\
\hline MEDNAX e-mail & $126(45)$ \\
\hline Twitter & $32(12)$ \\
\hline Facebook & $2(1)$ \\
\hline Other social media & $2(1)$ \\
\hline Other & $11(4)$ \\
\hline
\end{tabular}

Hospitals in 4 states averaged implementation of hospital restrictions as early as February and 3 states as late as April (Fig. 1b). The first international NICU restricted entry in January, followed by 21 units in March, and 5 in April. Overall, 184 (66\%) NICUs reported that their new policies during the COVID-19 pandemic were broadly more restrictive than the customary policies implemented during the winter influenza / respiratory syncytial virus season.

Changes in overall hospital entry screening policies became widespread during the COVID-19 pandemic (Table 2). These included significant increases in physical temperature checks as well as screening questions regarding travel history, fever, and illness for hospital entry.
Hospitals implemented parallel changes affecting NICU entry after the emergence of COVID-19 (Table 2). NICUs broadly implemented screening questions for travel history (76.5\%), fever and illness (94.9\%) as well as physical temperature checks $(72.2 \%)$. The number of NICUs allowing 24-h parental presence in the NICU decreased significantly $(83-53 \%, p<0.001)$. While full NICU team (e.g. nurse, doctor, nurse practitioner, RT etc) plus parental participation in NICU rounds predominated prior to the COVID-19 pandemic, this became significantly less common during the pandemic (71.1 vs. $32.1 \%, p<0.001$, Table 2). The number of states with over $80 \%$ of responding NICUs allowing full team and parental participation in NICU rounds fell from 20 (50\%, Fig. 1c) to 3 (8\%, Fig. 1d).

Within the 130 NICUs that restricted parental presence during the COVID-19 pandemic, we found a variety of policy measures described (Fig. 3). This included 7 NICUs (5\%) excluding all parental presence, 2 (2\%) of which would not allow parents to enter even with their infant in extremis. Most NICUs restricting parental presence only allowed one parent at the bedside at any time (85\%) and a minority $(25 \%)$ required families to choose a single parent to be allowed into the NICU for the entire hospital stay.

We examined the influence of NICU design (all open bay, single-family room, or hybrid) on NICU entry and NICU rounding policies. All NICU design types implemented significant increases in routine screening measures after the COVID-19 outbreak and had significant decreases in 24-h parental presence, but these restrictions were less in single-family room NICUs (84-64\%, absolute difference $20 \%, p=0.009)$ compared to hybrid $(91-57 \%$, absolute difference $34 \% p<0.001)$ and open bay units $(78-45 \%$, absolute difference $33 \%, \mathrm{p}<0.001$, Table 3). While 24-h unrestricted parental presence was not significantly different between all NICU designs prior to the COVID-19 pandemic, we found significant differences during the pandemic (single-family room $65 \%$, hybrid-design $57 \%$, open bay design $45 \%, p=.018$ ).

With respect to parental presence during rounds, prior to the emergence of COVID-19, we found significant differences in NICUs allowing full parental presence in rounds based on NICU design (SFR 83\%, hybrid NICUs 73\%, open bay NICUs $63 \%, p=0.013$ ). However, all NICU design types imposed a significant restriction in parental participation in NICU rounds during the pandemic (Table 3). After the emergence of COVID-19 we found no significant differences in full parental participation in NICU rounds across design types (SFR 37\%, hybrid 34\%, open bay $28 \%, p=0.6$ ). For the purpose of this research, we define the following NICU architectural designs: Open Bay - $90 \%$ or more of beds in rooms of 4, Hybrid - 11-89\% of beds in single patient rooms, and Single Family Room (SFR)- $90 \%$ or more of beds in single patient rooms. 
a) The Number of NICUs Represented From Each State

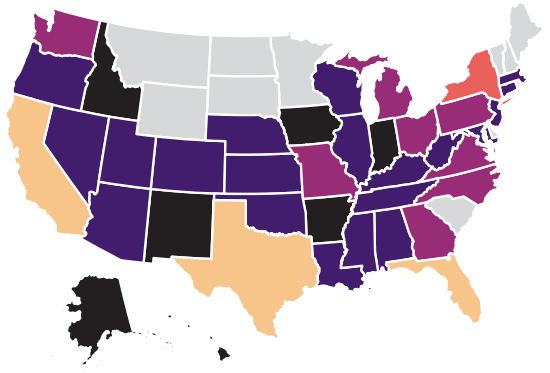

c) Allow Full Parental Participation in Rounds Pre-Covid-19

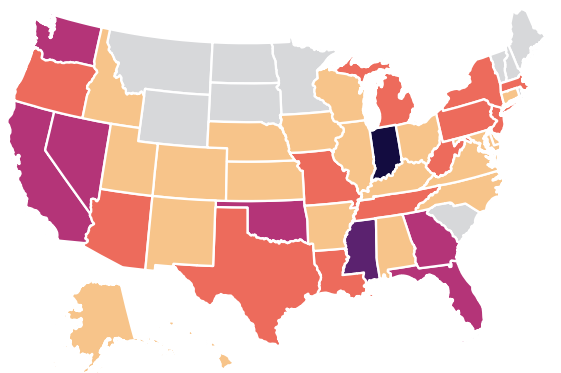

Fig. 1 Geographical distribution of responding NICUs, entry policy changes, and level of parental participation in rounds. Responses were received from NICUs in 40 out of 50 US States, with 3 states having more than 15 NICUs respond to the survey (a). The average date of changing hospital entry policies varied by state, with

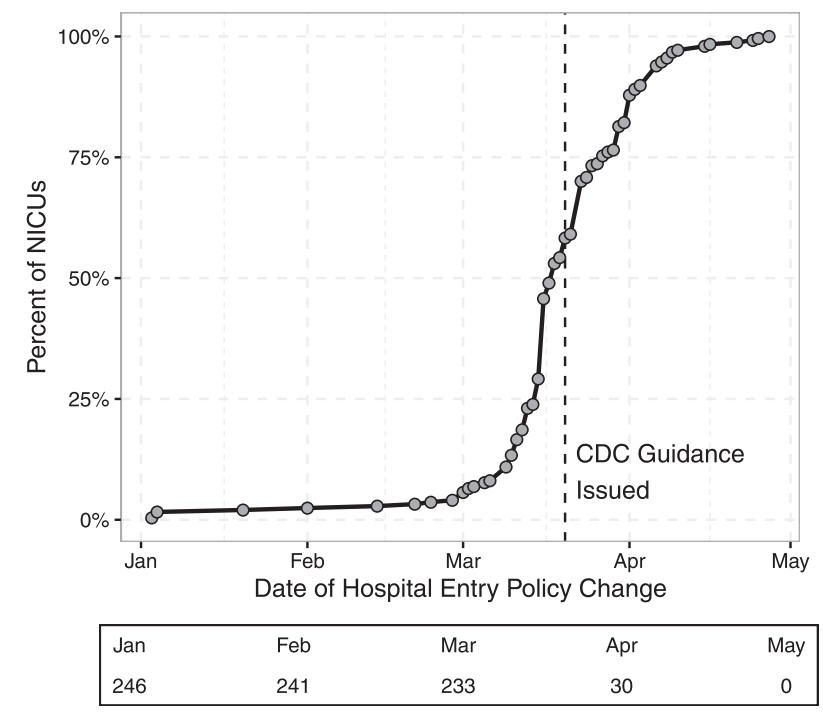

Fig. 2 The cumulative count of changes in hospital entry policies by calendar date. Very few hospitals altered their entry dates in January or February, 2020. However this rapidly changed in March with most NICUs changing their policy prior to the issuance of specific guidance from the Centers for Disease Control and Prevention (CDC) on March 20, 2020.

NICUs reported significant changes in staffing or outcome measures temporally associated with the COVID-19 pandemic. These included $28 \%$ of sites reducing nursing b) Average Date of Hospital Entry Policy Change

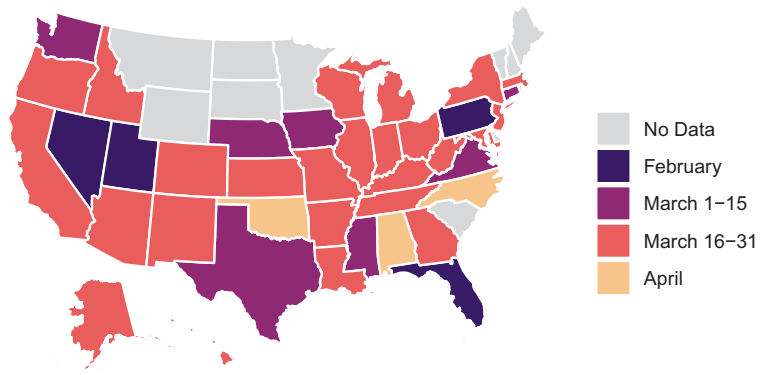

d) Allow Full Parental Participation in Rounds Post-Covid-19
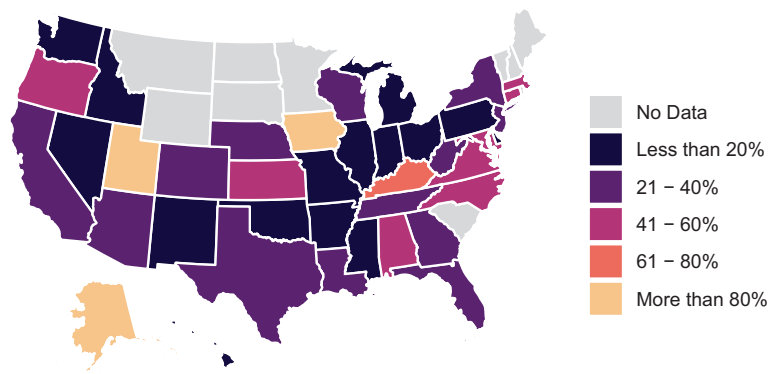

the majority averaging policy change between March 16 and 31 (b). Prior to the onset of the COVID-19 pandemic, permitting full parental participation in NICU rounds was commonplace $(\mathbf{c})$. However, during the pandemic very few states had a majority of NICUs allowing full parental participation in rounds.

staff hours, 28\% reducing physical (PT), occupational, and/ or speech therapy and $23 \%$ delaying non-urgent procedures (most commonly gastrostomy tubes). We also found $21 \%$ of NICUs reported decreased lactation consultant visits, $16 \%$ restricted donor milk utilization, and $8 \%$ reported changes in breastfeeding rates. In all, 120 (43\%) NICUs reported reductions in therapy services, lactation medicine, and/or social work support. Social services and lactation medicine offered supplemental telehealth support in 109 (41\%) NICUs.

\section{Discussion}

Prohibitions on family presence for critically ill or dying adults as a result of COVID-19 pandemic have been widely reported by the media. Little is known however regarding how COVID-19 has impacted family presence for minors, including the most vulnerable in our population-those admitted to NICUs. These data represent the first report documenting the widespread, rapid, and profound restrictions in hospital and NICU visitation practices secondary to the COVID-19 pandemic. Policy changes began in early January and hospitals rapidly adopted entry limitations throughout March 2020. The result has been a significant shift in family presence for sick infants and of the way 
Table 2 Hospital and NICU policy changes during the COVID-19 pandemic.

\begin{tabular}{lccc}
\hline & $\begin{array}{l}\text { Pre-COVID } \\
n=277 \\
\text { No. }(\%)\end{array}$ & $\begin{array}{l}\text { During COVID } \\
n=277 \\
\text { No. }(\%)\end{array}$ & $P$ value $^{\mathrm{a}}$ \\
& & & \\
\hline Hospital entry policies & $25(9)$ & $220(79)$ & $<0.001$ \\
Screening questions for travel history & $91(33)$ & $268(97)$ & $<0.001$ \\
Screening questions for fever and illness & $11(4)$ & $226(82)$ & $<0.001$ \\
Screening temperature check & & & $<0.001$ \\
NICU entry policies & $78(28)$ & $212(77)$ & $<0.001$ \\
Screening questions for travel history & $214(77)$ & $263(95)$ & $<0.001$ \\
Screening questions for fever and illness & $66(24)$ & $200(72)$ & $<0.001$ \\
Screening temperature check & $48(17)$ & $130(47)$ & $<0.001$ \\
Any restrictions on parental visitation & & $89(32)$ & $<0.001$ \\
NICU rounding policy & $197(71)$ & $114(41)$ & $<0.001$ \\
Full team and parental participation & $33(12)$ & $74(27)$ & \\
Limited team and parental participation & $47(17)$ & & \\
No formal procedure or other procedure & &
\end{tabular}

${ }^{\mathrm{a}}$ False discovery rate adjusted $p$ value.

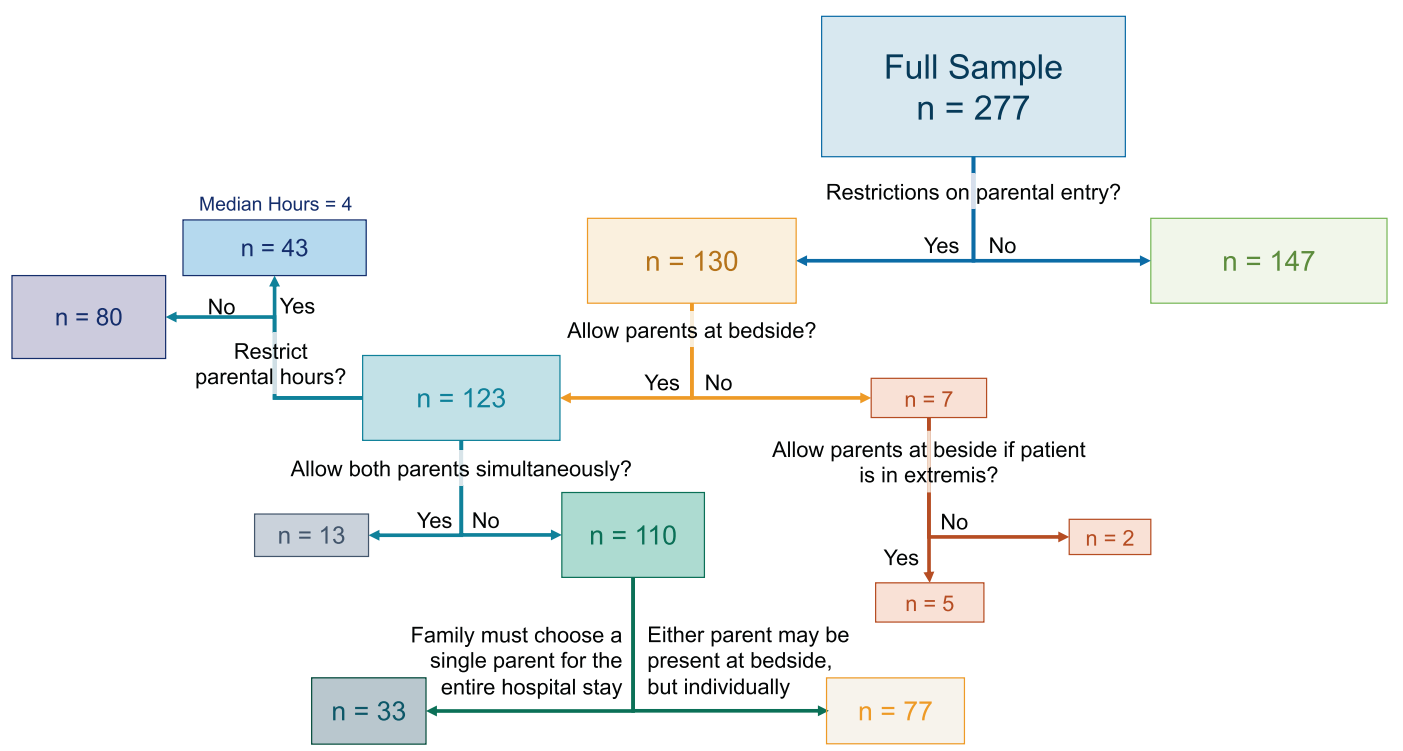

Fig. 3 Flow diagram of restrictions to parental presence in the NICU after the emergence of COVID-19. Overall, 130 of 277 NICUs (47\%) had restrictions to NICU parental in the NICU during the Covid-19 pandemic. Of these, most allowed only a single parent at

parents experience the first phases of their newborn's lives in the NICU environment. An additional secondary effect of COVID-19 has been delays in elective procedures and the reduction of staff in many NICUs including therapy services, lactation support, and social services-all important for optimal outcomes. Ultimately, as health care systems attempt to prevent the spread of coronavirus, new policies have led to families interacting very differently with their infants in the NICU—or not at all. These rapidly instituted the bedside and 33 NICUs required families to choose a single parent for the entire hospital stay. Prohibition of parental presence in the NICU was rare but occurred at 7 sites $(3 \%)$

changes may carry with them the risk of secondary unintended consequences [16].

In this cross-sectional survey of 277 NICUs we found widespread implementation of hospital and NICU screening measures, including decreases in NICUs allowing 24-h parental presence (83-53\%). Single-family room NICU design may attenuate some restrictions. Private rooms make certain infection control measures easier by providing physical distancing, physical barriers, and separate air supplies [17, 18]. 
Table 3 The effect of NICU design on the changes in NICU entry and rounding policies.

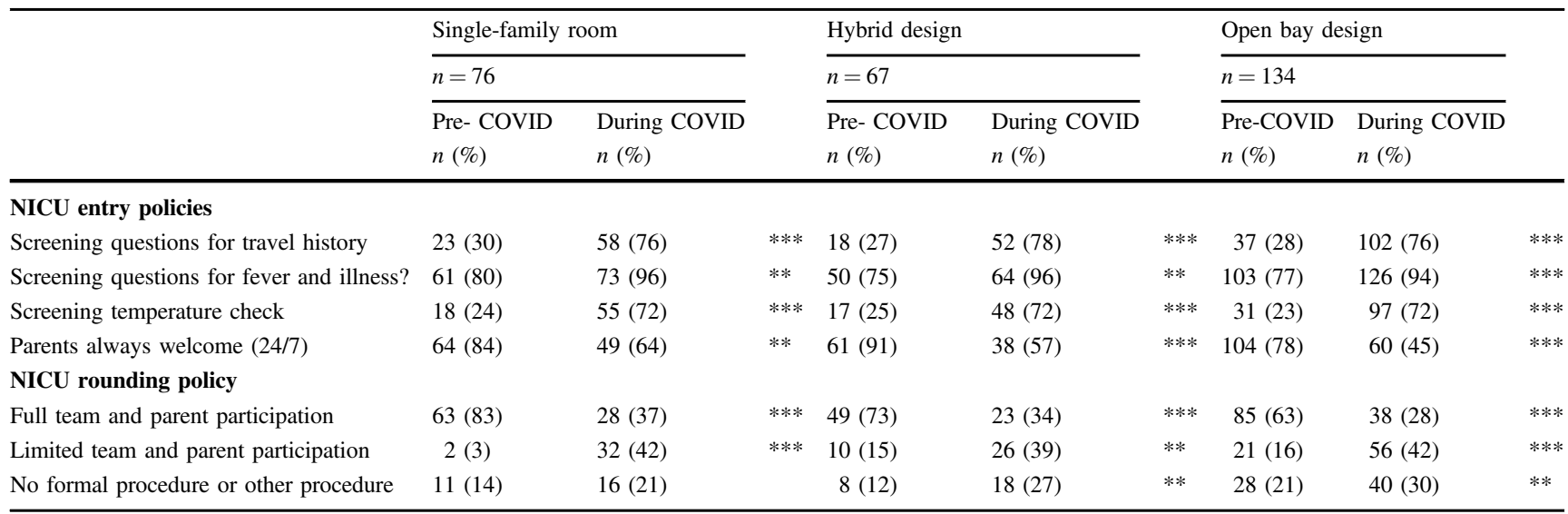

False discovery rate adjusted $p$ values

NICU neonatal intensive care unit.

$* * * p<0.001, * * p<0.01, * p<0.05$.

At baseline we found no differences in 24-h parental presence among various NICU designs. However, we found more NICUs with single-family room design able to maintain 24-h parental presence and interaction with babies and caregivers than open bay units (64 vs. $45 \%$ ). This clinically meaningful preservation of parental presence occurred at a time when many other limitations were imposed, including limiting parental participation in NICU rounds.

We find that restrictions in NICU parental presence have been widely adopted and that the ability for parents to participate in aspects of shared decision making such as family-centered rounds have diminished significantly. Family-centered rounds (FCRs) are multidisciplinary rounds that occur at the patient's bedside, with family and caregivers integrated into the clinical decision making. Prior to the COVID-19 pandemic, $71 \%$ of NICUs allowed full team and parental participation in NICU rounds, falling to $32 \%$ during the pandemic. Family-centered care has been recommended by both the Institute of Medicine [19] and AAP [20] as key to improving the quality and safety of health care. Recommendations include FCRs to ensure that decisions on the plan of care incorporates family involvement in decision making [20]. Identified benefits of FCR include improved "patient satisfaction, communication, discharge planning, medical education, and patient safety" [21]. It has also been suggested that family involvement in care can decrease family stress while improving patient's outcomes [22, 23]. The abrupt restrictions in parental presence and family participation in rounds necessarily also disrupts the ability to provide family-centered care. The impact of these changes on parental stress, patient safety, and patient outcomes require further investigation.

Prior infectious outbreaks, such as Severe Acute Respiratory Syndrome, resulted in increased rates of mental health disorders, including depression, anxiety as well as posttraumatic stress disorder [24]. Data from Wuhan, China demonstrates a similar increase in psychopathology, with some reports suggesting that women may have an increased burden of mental health disorders in the current pandemic $[25,26]$. Pregnancy has known associations with significant emotional distress under normal circumstances, and women's mental health in the perinatal period is significantly affected by access to social support and resources [27, 28]. This raises the concern that pregnant and postpartum women may be particularly vulnerable to the psychosocial hardships caused by the pandemic. Studies have also "suggested that higher maternal stress may lead to reductions in warm, contingent caregiving (i.e., caregiver responsiveness), which in turn can affect infant neurodevelopment" [29-31]. The described restrictions on parental presence for ill newborns may further magnify the existing stressors associated with the perinatal period and NICU admission.

An important component of the care for ill newborns, especially those born prematurely, is the developmental team, whose members include disciplines such as OT, PT, and ST with involvement from social work, and lactation consultants [32, 33]. Overall, 43\% of responding NICUs reported decreases in at least one area of these support services. While some sites offered telehealth options for these services, the efficacy of telehealth in this setting is unknown. In addition to direct patient care, the developmental team plays an important role in educating parents on key techniques to implement on a daily basis both in the hospital and post-discharge [34, 35]. While possibly implemented in an effort to minimize the spread of COVID19 to vulnerable patients, diminished social work, and developmental care services to premature infants may have long-term adverse consequences. 
Strengths of this study include responses from a large number of hospitals and NICUs across the United States and around the world. These data are sufficient to provide a granular understanding of the timeline by which hospitals and facilities implemented restrictions and how access became significantly limited even in areas of the hospital with minimal disease burden from COVID-19 such as the NICU. Further, many facilities provided data regarding limitations in support services, providing indications of the secondary effects the COVID-19 pandemic has quickly caused. Limitations include those inherent to survey studies including inability to verify responses, incomplete responses, and inability to ask follow-up questions of respondents. In addition, despite our stated intention to receive responses from NICU medical or nursing directors, the identity of the respondent could not be controlled for. We cannot rule out further evolution of hospital policies after the completion of the survey window. Lastly, due to the distribution platforms used for this survey, a denominator and thus response rate could not be determined.

\section{Conclusion}

In summary, we report dramatic and rapid changes in hospital and NICU entry policies related to COVID-19. These changes have significantly affected parental presence for NICU admitted neonates, although single-family room design may partially attenuate this effect. In addition to these restrictions, limitations in support staff as well as delays in elective procedures for NICU admitted infants are common. In total, the rapid implementation of these sweeping changes may have substantial impact on parental and family well-being and may lead to detrimental effects on neonatal health outcomes. Further investigation regarding the short- and long-term impact of these policy changes is urgently needed.

Acknowledgements We thank Dr. Veeral Tolia for his critical review of this manuscript prior to journal submission.

Funding This article is published as part of a supplement sponsored by Philips.

\section{Compliance with ethical standards}

Conflict of interest The Methodist Healthcare System IRB approved this research. RDW has received consulting fees from Philips HealthTech, owns equity in Mednax, and received lecture fees from $\mathrm{P}+\mathrm{G}$ (Pampers). RDW also holds patents for two products (not for sale). The remaining authors have declared no competing interests.

Ethics The Methodist Healthcare System IRB approved this research.

Publisher's note Springer Nature remains neutral with regard to jurisdictional claims in published maps and institutional affiliations.

\section{Appendix: COVID visitation questionnaire}

\section{NICU demographics}

1. Level: (1-4)

- 1

- 2

- 3

- 4

2. Design:

- "Open Bay" - 90\% or more of beds in rooms of 4

- "Hybrid" - 11-89\% of beds in single patient rooms

- "Single Family Room" - 90\% or more of beds in single patient rooms.

3. \# of NICU beds

4. Are you in the United States?

- Yes

a) State

b) zip code

- No

a) City

b) Country

5. Which description accurately describes your unit?

- NICU, out born only (typically a children's hospital with no delivery service in-house)

- NICU, inborn only

- NICU with both inborn and transported admissions

6. Where did you find out about the survey? American Academy of Pediatrics e-mail

- American Academy of Pediatrics e-mail

- Mednax e-mail

- Twitter

- Facebook

- Other social media

- Other

a) Where did you hear about the survey?

\section{Visiting policies}

7. When did your hospital/NICU change its visiting policy?

8. What was your Pre-COVID NICU visiting policy?

- Screening question(s) for travel history?
a) Yes
b) No

- Screening question (s) for fever or illness?

a) Yes

b) No 
- Screening temperature check?
a) Yes
b) No

- Parents and visitors always welcome (24/7)?
a) Yes
b) No

- Parents always welcome; some restrictions (number, time of day) on other visitors?
a) Yes
b) No

- Restrictions on parent visitation (e.g., limited visiting hours)
a) Yes
b) No

- If you have additional visiting polies please briefly explain

- What was your Pre-COVID NICU rounding policy

a) Full team and parent participation at the bedside

b) Full team and parent participation away from the bedside

c) Limited team and parent participation (e.g., nurse, RT; parent not present except under special circumstances)

d) No formal daily rounding procedure

e) other

9. What was your Pre-COVID hospital-wide visiting policy?

- Screening question(s) for travel history?
a) Yes
b) No

- Screening question(s) for fever or illness?
a) Yes
b) No

- Screening temperature check?
a) Yes
b) No

- Parents /spouses and visitors always welcome?
a) Yes
b) No

- Parents /spouses always welcome; some restrictions (number, time of day) on other visitors?
a) Yes
b) No

- Restrictions (limited visiting hours) on immediate family visitation (e.g., spouse, parent)
a) Yes
b) No

10. What is your current COVID NICU visiting policy?

- Screening question(s) for travel history?
a) Yes
b) No

- Screening question(s) for fever or illness?
a) Yes
b) No

- Screening temperature check?
a) Yes
b) No

- Do you have any restrictions on parent visitation? (e.g., limited visiting hours)
a) Yes (e.g., limited visiting hours)
b) No (24/7 visitation)

- Are parents completely restricted from the bedside?
a) Yes
b) No

- If a patient is in extremis, are parents allowed at the bedside?
a) Yes
b) No

- Is only one parent allowed at the bedside at a time?
a) Yes
b) No

- Are you restricting to just one chosen parent allowed to visit the entire hospital stay? (i.e., one parent cannot physically visit at all)?
a) Yes
b) No

- Can parents switch off visitation within the same day?
a) Yes
b) No

- Can parents switch off visitation only across different days?
a) Yes
b) No

- Are both parents allowed at the bedside simultaneously?
a) Yes
b) No

- Are there restrictions on the number of hours per day at the bedside?
a) Yes
b) No

- How many hours a day are parents allowed at the bedside?

- What is your current COVID NICU rounding policy?

a) Full team and parent participation at the bedside

b) Full team and parent participation away from the bedside 
c) Limited team and parent participation (e.g., nurse, RT; parent not present except under special circumstances)

d) No formal daily rounding procedure

e) Other

- Do you have additional visiting policies we haven't asked about, please briefly describe.

11. What is your current COVID hospital-wide visiting policy?

- Screening question(s) for travel history?
a) Yes
b) $\mathrm{No}$

- Screening question(s) for fever or illness?
a) Yes
b) $\mathrm{No}$

- Screening temperature check?
a) Yes
b) No

- Parents /spouses and visitors always welcome? (24/7)
a) Yes
b) No

- Parents /spouses always welcome; some restrictions (number, time of day) on other visitors?
a) Yes
b) No

- Restrictions (limited visiting hours) on immediate family visitation (e.g., spouse, parent)
a) Yes
b) $\mathrm{No}$

\section{Flu season visitation policy}

12. Did you have a flu/RSV season policy that differed from your regular NICU visiting policy?

- Yes

a) the same as the COVID visitation policy?

1. Yes

2. No

b) is the flu/RSV policy less restrictive than the COVID visitation policy?
1. Yes
2. No

- No

Have you seen any detectable change in any outcome measures listed below? If yes, Outcome Measures
Please check yes. If data is available, please insert. If no, or unsure at this point, select no change.

\begin{tabular}{l}
\hline Outcomes measure \\
\hline Parental satisfaction scores (Q4 2019 No $\quad$ Supporting Data \\
vs. Q1-2 of 2020) \\
Are parents making more use of their \\
allotted/restricted visiting time? (i.e., are \\
they visiting more days than prior to the \\
COVID visiting changes). \\
Staff turnover \\
How many staff have resigned or \\
reduced hours since March 12020 ? \\
RN vs. MD vs. NP vs. RT? \\
Nosocomial infection (Q4 2019 vs. Q1- \\
2 of 2020) \\
Breast Feeding rates? \\
Have your tightening the criteria around \\
donor breast milk? \\
Have you had a decrease in ventilator \\
days? (i.e., in an effort to free up \\
ventilators for adults) \\
Has your length of stay decreased? \\
Have your PPD scores for mothers \\
increased (Edinburgh scale)? \\
Have infants experienced a delays in \\
non-urgent procedures? (i.e G-tube \\
placement etc.) \\
Do you have less ancillary services \\
available (less OT/PT/Speech) January \\
2020 vs. March 2020? \\
Do you have less Lactation visits \\
January 2020 vs. March 2020? \\
Do you have less Social work visits \\
January 2020 vs. March 2020? \\
Are social work/lactation providing \\
Televisiting/support for families? \\
\hline
\end{tabular}

Short answer:

13. Assuming policies in your unit are more restrictive than in non-pandemic times, have you increased your support staff to account for more phone calls and updates for families?

14. How have you implemented CDC social distancing guidelines for parents/caregivers that are allowed into the UNIT?

15. How have you implemented CDC social distancing guidelines for healthcare workers?

16. How have your changed your practice around HCW that also service adult patients (i.e., radiology, RTs, phlebotomy).

17. Suggestions for the future if you had to do it again

Appendix Legend: The questionnaire was entered into REDCap with branching logic incorporated as appropriate. 


\section{References}

1. United States Department of Health and Human Services (US DHHS), Centers for Disease Control and Prevention (CDC), National Center for Health Statistics (NCHS), Division of Vital Statistics, Natality public-use data 2016-2018, on CDC WONDER Online Database, September 2019. http://wonder.cdc.gov/na tality-expanded-current.html

2. Baley J. Committee on fetus and newborn. Skin-to-skin care for term and preterm infants in the neonatal ICU. Pediatrics. 2015;136:596-9.

3. Conde-Agudelo A, Díaz-Rossello JL. Kangaroo mother care to reduce morbidity and mortality in low birthweight infants. Cochrane Database Syst Rev. 2016. https://doi.org/10.1002/ 14651858.cd002771.pub4

4. Welch M, Firestein M, Austin J, Hane A, Stark R, Hofer M, et al. Family Nurture Intervention in the Neonatal Intensive Care Unit improves social-relatedness, attention, and neurodevelopment of preterm infants at 18 months in a randomized controlled trial. J Child Psychol Psychiatry. 2015;56:1202-11. https://doi. org/10.1111/jcpp. 12405

5. Lee L, Carter M, Stevenson S, Harrison H. Improving familycentered care practices in the NICU. Neonatal N. 2014;33: $125-32$.

6. Gooding JS, Cooper LG, Blaine AI, Franck LS, Howse JL, Berns SD. Family support and family-centered care in the neonatal intensive care unit: origins, advances, impact. Semin Perinatol. 2011;35:20-28. https://doi.org/10.1053/j.semperi.2010.10.004

7. Interim Guidance for Childcare Programs and K-12 Schools. Centers for Disease Control and Prevention. 2020. https://www. cdc.gov/coronavirus/2019-ncov/community/schools-childcare/ guidance-for-schools.html. Accessed 29 April 2020.

8. Centers for Disease Control and Prevention. Steps healthcare facilities can take now to prepare for COVID-19. 2020. https://www.cdc.gov/coronavirus/2019-ncov/hcp/steps-to-prepare. html. Accessed 29 April 2020.

9. Centers for Disease Control and Prevention. Social distancing, quarantine, and isolation. 2020. https://www.cdc.gov/coronavirus/ 2019-ncov/prevent-getting-sick/social-distancing.html. Accessed 29 April 2020.

10. Huang L, Zhang X, Zhang X, Wei Z, Zhang L, Xu J, et al. Rapid asymptomatic transmission of COVID-19 during the incubation period demonstrating strong infectivity in a cluster of youngsters aged 16-23 years outside Wuhan and characteristics of young patients with COVID-19: a prospective contact-tracing study. J Infect. 2020;80. https://doi.org/10.1016/j.jinf.2020.03.006

11. Ye F, Xu S, Rong Z, Xu R, Liu X, Deng P, et al. Delivery of infection from asymptomatic carriers of COVID-19 in a familial cluster. Int J Infect Dis. 2020;94:133-8. https://doi.org/10.1016/j. ijid.2020.03.042

12. Dunn A. Fact check: are coronavirus patients dying alone in hospitals? USA Today. 2020. https://www.usatoday.com/story/ news/factcheck/2020/04/09/fact-check-coronavirus-patients-dying-a lone-hospitals/5114282002/. Accessed 14 May 2020.

13. Hafner K. 'A heart-wrenching thing': hospital bans on visits devastate families. The New York Times. 2020. https://www. nytimes.com/2020/03/29/health/coronavirus-hospital-visit-ban.html. Accessed 14 May 2020.

14. Benjamini Y, Hochberg Y. Controlling the false discovery rate: a practical and powerful approach to multiple testing. J R Stat Soc Ser B Stat Methodol. 1995;57:289-300.

15. Kates, J, 2020. Stay-at-home orders to fight COVID-19 in the United States: the risks of a scattershot approach. KFF. https://www.kff.org/coronavirus-policy-watch/stay-at-home-ordersto-fight-covid19/ Accessed 20 May 2020.

16. Virani AK, Puls HT, Mitsos R, Longstaff H, Goldman RD, \& Lantos JD. Ethics rounds: benefits and risks of visitor restrictions for hospitalized children during the COVID pandemic. Pediatrics. 2020. https://doi.org/10.1542/peds.2020-000786

17. Taylor E, Card AJ, Piatkowski M. Single-occupancy patient rooms: a systematic review of the literature since 2006. HERD. 2018;11:85-100.

18. O'Callaghan N, Dee A, Philip RK. Evidence-based design for neonatal units: a systematic review. Matern Health Neonatol Perinatol. 2019;5. https://doi.org/10.1186/s40748-019-0101-0

19. Institute of Medicine. Crossing the quality chasm: a new health system for the 21st century. Washington, DC: National Academy Press; 2001.

20. Committee on Hospital Care and Institute for Patient and Family Centered Care. Patient and Family centered care and the pediatrician's role. Pediatrics. 2012;129:394-404.

21. Mittal V. Family-centered rounds. Pediatr Clin North Am. 2014;61:663-70. https://doi.org/10.1016/j.pcl.2014.04.003

22. Melnyk BM, Feinstein NF. Reducing hospital expenditures with the COPE (creating opportunities for parent empowerment) program for parents and premature infants. Nurs Admin Q. 2009;33:32-37. https://doi.org/10.1097/01.naq.0000343346. 47795.13

23. Davidson J, Powers K, Hedayat K, Tieszen M, Kon A, Shepard E, et al. Clinical practice guidelines for support of the family in the patient-centered intensive care unit: American College of Critical Care Medicine Task Force 2004-2005. Crit Care Med. 2007;35:605-22. https://doi.org/10.1097/01.ccm.0000254067.14607.eb

24. Ko CH, Yen CF, Yen JY, Yang MJ. Psychosocial impact among the public of the severe acute respiratory syndrome epidemic in Taiwan. Psychiatry Clin Neurosci. 2006;60:397-403.

25. Liu N, Zhang F, Wei C, Jia Y, Shang Z, Sun L, et al. Prevalence and predictors of PTSS during COVID-19 outbreak in China hardest-hit areas: Gender differences matter. Psychiatry Res. 2020;287:112921 https://doi.org/10.1016/j.psychres.2020.112921

26. Cao W, Fang Z, Hou G, Han M, Xu X, Dong J, et al. The psychological impact of the COVID-19 epidemic on college students in China. Psychiatry Res 2020;287:112934 https://doi.org/10. 1016/j.psychres.2020.112934

27. Franks WLM, Crozier KE, Penhale BLM. Women's mental health during pregnancy: a participatory qualitative study. Women Birth. 2017;30:e179-187.

28. Katz J, Crean HF, Cerulli C, Poleshuck EL. Material hardship and mental health symptoms among a predominantly low income sample of pregnant women seeking prenatal care. Matern Child Health J. 2018;22:1360-7.

29. Rallis S, Skouteris H, McCabe M, Milgrom J. A prospective examination of depression, anxiety and stress throughout pregnancy. Women Birth. 2014. https://www.sciencedirect.com/ science/article/pii/S1871519214000821

30. Ursache A, Merz EC, Melvin S, Meyer J, Noble KG. Socioeconomic status, hair cortisol and internalizing symptoms in parents and children. Psychoneuroendocrinology. 2017;78:142-50. https://doi.org/10.1016/j.psyneuen.2017.01.020

31. Troller-Renfree, A, Brito, N, Desai, P, Leon-Santos, A, Wiltshire, C, Motton, S, et al., Infants of mothers with high physiological stress show alterations in brain function. Dev Sci. 2020. https:// doi.org/10.1111/desc. 12976

32. Barbosa V. Teamwork in the neonatal intensive care unit. Phys Occup Ther Pediatr. 2013;33:5-26. https://doi.org/10.3109/ 01942638.2012.729556 
33. Macho P. Individualized developmental care in the NICU: a concept analysis. Adv Neonatal Care 2017;17:162-74. https://doi. org/10.1097/ANC.0000000000000374

34. Ross K, Heiny E, Conner S, Spener P, Pineda R. Occupational therapy, physical therapy and speech-language pathology in the neonatal intensive care unit: Patterns of therapy usage in a level IV
NICU. Res Dev Disabil. 2017;64:108-17. https://doi.org/10.1016/ j.ridd.2017.03.009

35. Borges Nery P, Snider L, Camelo J, Boychuck Z, Khodary F, Goldschleger J, et al. The role of rehabilitation specialists in Canadian NICUs: a 21st century perspective. Phys Occup Ther Pediatr. 2018;39:33-47. https://doi.org/10.1080/01942638.2018.1490846 